\title{
An Interactive Folktale System and an Authoring System That Supports Its Realization
}

\author{
Ryohei Nakatsu, Yurika Nagai, Kozi Miyazaki, Yurika Nagai, and Takenori Wama \\ Kwansei Gakuin University, School of Science and Technology \\ 2-1 Gakuen, Sanda 669-1337, Japan
}

\begin{abstract}
Along with the advancement of three dimensional computer graphics technology and interaction technology, new types of education system as well as entertainment system are expected to emerge. In this paper as one example of such a system, we propose "Interactive Folktale System," This system makes it possible for people, especially children, to enjoy folktales in various ways. They can appreciate the regeneration of original tales and its variations. Also they can interact with the story, by communicating with the characters in the story or by playing a role of one of the characters. Moreover, they can easily create a new folktale by changing the original story or by describing a script for the new story. Also the architecture of an authoring system that supports that realization of the interactive folktale system is described.
\end{abstract}

